\title{
Use of proton pump inhibitors and $\mathrm{H} 2$ blockers and risk of pneumonia in older adults: a population-based case-control study
}

Sascha Dublin, MD, PhD ${ }^{1,2}$, Rod L. Walker, MS ${ }^{1}$, Michael L. Jackson, MPH, PhD ${ }^{1}$, Jennifer C. Nelson, $\mathrm{PhD}^{1,3}$, Noel S. Weiss, MD, DrPH ${ }^{2}$, and Lisa A. Jackson, MD, MPH ${ }^{1,2}$

${ }^{1}$ Group Health Research Institute, Seattle, Washington

2 Department of Epidemiology, University of Washington, Seattle, Washington

${ }^{3}$ Department of Biostatistics, University of Washington, Seattle, Washington

\section{Abstract}

Purpose-To examine whether use of proton pump inhibitors (PPIs) and $\mathrm{H} 2$ blockers is associated with increased pneumonia risk.

\begin{abstract}
Methods-We conducted a population-based, nested case-control study within Group Health, an integrated healthcare delivery system. Among community-dwelling, immunocompetent adults aged 65-94, we identified presumptive cases of ambulatory and hospitalized community-acquired pneumonia in 2000-2003 from ICD-9 codes and validated them by medical record review $(\mathrm{N}=1125)$. Controls were selected, matched to cases on age, sex, and calendar year $(\mathrm{N}=2235)$. Current PPI or $\mathrm{H} 2$ blocker use was ascertained from computerized pharmacy records. Comorbid illnesses and other characteristics were ascertained by medical record review. Multivariable conditional logistic regression was used to examine the association between medication use and pneumonia risk. We conducted sensitivity analyses using only administrative and pharmacy data to assess how these results differed from our primary results.
\end{abstract}

Results-The prevalence of PPI or H2 blocker use was 21\% (241/1125) for pneumonia cases and 16\% (350/2235) for controls (adjusted odds ratio [OR] 1.03, 95\% CI 0.86-1.24, compared to nonuse). No increased risk was seen for recent initiation. The prevalence of PPI use was $12 \%$ (132/1125) for cases and 7\% (160/2235) for controls (adjusted OR 1.13, 95\% CI 0.88-1.44). Analyses using only administrative and pharmacy data yielded risk estimates farther from the null (adjusted OR 1.32, 95\% CI 1.17-1.49, for current PPI use versus nonuse).

Conclusions-Use of PPIs and $\mathrm{H} 2$ blockers is not associated with increased pneumonia risk in older adults. The increased risk observed in some prior studies may reflect confounding.

\section{Keywords}

pneumonia; acid-suppressing medications; proton-pump inhibitors; H2 blockers; case-control studies

Correspondence and requests for reprints: Sascha Dublin, MD, PhD; Group Health Research Institute; 1730 Minor Avenue, Suite 1600; Seattle, WA 98101-1448. Phone 206-287-2870; Fax 206-287-2871., dublin.s@ghc.org.

Conflict of Interest: Jennifer C. Nelson has done consulting work for Glaxo Smith Kline. 


\section{Introduction}

Acid-suppressing medications (proton pump inhibitors [PPIs] and histamine-type 2 receptor blockers [H2 blockers]) are widely used for such conditions as gastroesophageal reflux, dyspepsia, and peptic ulcer and are often used for long periods of time. An estimated 7 to 8 million prescriptions for PPIs were dispensed each month in the United States in 2004. ${ }^{1}$ A substantial proportion of use is for conditions for which these medications are not approved by the United States Food and Drug Administration or for which there is little evidence of benefit. ${ }^{2-8}$

Acid-suppressing medications could plausibly increase pneumonia risk. They increase gastric $\mathrm{pH}$, allowing gastric colonization with bacteria that if aspirated could cause pneumonia. ${ }^{9-11}$ Randomized controlled trials (RCTs) of these medications provide little relevant information because most studies had small sample size and short duration and did not actively elicit respiratory illnesses as an outcome. ${ }^{12}$ Two studies, a meta-analysis and a pooled analysis, have summarized the results of multiple RCTs (Table 1). ${ }^{12,}{ }^{13}$ In both, risk estimates for the association of PPI use with pneumonia had wide confidence intervals, consistent either with no association or with substantial harm. Results from observational studies are conflicting (Table 1). In several studies, pneumonia risk was 30-80\% higher in current users of PPIs compared to nonusers. ${ }^{14-17}$ Another study observed no overall increased risk with PPI use but a 7-fold increased risk of pneumonia in the first 2 days of initiating either $\mathrm{H} 2$ blocker or PPI use, an elevation that declined with continuing use. ${ }^{18}$ This pattern is not consistent with the principal mechanism by which these medications are hypothesized to increase pneumonia risk but instead may reflect bias, for example if an acidsuppressing medication were started at the time of an acute change in health status (e.g. following an invasive procedure or initiation of chemotherapy) or to treat early symptoms of pneumonia.

Prior observational studies, which relied on administrative data, had important limitations. Most did not validate pneumonia cases, and none reviewed records to identify potential confounding factors such as comorbidity and functional and cognitive status. Many pneumonia risk factors, including chronic lung and heart disease, are poorly measured in administrative data. ${ }^{19},{ }^{20}$ We examined the association between PPI and $\mathrm{H} 2$ blocker use and risk of community-acquired pneumonia (CAP) using data from a previously-conducted study in which all pneumonia cases were validated and detailed information was collected from medical records about potential confounding factors. ${ }^{21}$ Our aim was to test the hypothesis that use of PPIs and $\mathrm{H} 2$ blockers is associated with increased CAP risk.

\section{Methods}

\section{Overview and Setting}

We analyzed data from a nested case-control study previously conducted at Group Health $(\mathrm{GH})$, an integrated healthcare delivery system in Washington State, USA. GH maintains extensive paper and electronic medical records with information about inpatient stays and ambulatory visits and also maintains electronic laboratory and pharmacy data. The aim of the original study was to examine pneumonia risk in relation to receipt of influenza vaccine. ${ }^{21}$ We relied on the original study's population, ascertainment of outcomes, and covariate information and supplemented these data with additional information about use of PPIs and H2 blockers. This research was approved by the Group Health Human Subjects Review Committee with a waiver of consent. 


\section{Source population}

The study population consisted of GH members who were age 65-94 with at least 2 years of continuous GH membership, community-dwelling, and not immunosuppressed.

Immunosuppression was defined as having serious cancer, recent cancer treatment, or severe kidney disease, or receiving certain immunosuppressive medications or medications for human immunodeficiency virus (Appendix Table 1). Study eligibility was determined as of September 1 of 2000, 2001 or 2002 (per the aims of the original study) based on computerized pharmacy, laboratory, and encounter data and confirmed by medical record review. The goal of restricting the study population was to decrease potential confounding by health conditions that might affect both medication use and risk of pneumonia.

\section{Selection of cases and controls}

The methods used to validate pneumonia cases have been described previously. ${ }^{22}$ Briefly, we identified presumptive cases using International Classification of Disease, version 9 (ICD-9) codes from inpatient and outpatient encounters and validated them through review of chest radiograph reports and hospitalization records. Cases were judged to have pneumonia if the chest radiograph showed a parenchymal infiltrate not known to be chronic or, for hospitalized cases, if the final physician assessment was that pneumonia caused the illness present at admission. Cases of hospital-acquired pneumonia or massive aspiration were excluded. Cases were identified from September 1 until the end of influenza season in each study year, based on the aims of the original study. For people with multiple episodes of pneumonia, we included only the first episode. Figure 1 shows the flow of potential participants through the study. Cases were assigned an index date which was the date of pneumonia diagnosis, defined as the date of the first positive chest radiograph for cases validated through radiology reports or the date of admission for cases validated from hospital records.

Up to 2 controls were selected for each case matched on age, sex, and calendar year. Controls were required to have survived, remained pneumonia-free, and remained enrolled in GH through the index date of their matched case (incidence density sampling) and were assigned the case's index date.

\section{Measures of drug exposure and pneumonia risk factors}

GH's pharmacy database provided information on prescription fills. In prior studies, $98 \%$ of GH members in this age group have reported filling all or almost all of their prescriptions at GH pharmacies. ${ }^{23}$ Pharmacy records include a unique patient identifier, medication name, strength, route of administration, date dispensed, quantity dispensed, and days supplied. H2 blockers became available over the counter (OTC) in the U.S. in 1995, while PPIs became available in the fall of 2003. Thus, H2 blockers were available OTC during our study period, but PPIs were not. OTC medications are available for purchase at GH pharmacies, and data about these purchases are included in GH's pharmacy database. In 2000, Boudreau et al. surveyed GH members about use of medications and compared their responses with pharmacy data. ${ }^{24}$ Among older adults with a drug benefit, the prevalence of use of acidsuppressing medications was the same from both sources (18\%). For those without a drug benefit, the prevalence was $9 \%$ from pharmacy data and $12 \%$ from survey data. These data indicate that at the time of our study, the majority of use of these medications was captured in $\mathrm{GH}$ pharmacy data.

For these analyses, only oral medications were included. We defined "current use" of a PPI or $\mathrm{H} 2$ blocker as having filled 2 or more prescriptions with at least 30 days' supply within the 180 days prior to index date. "Possible use" was defined as filling at least one prescription in the 365 days prior to index date but not meeting criteria for current use. 
"Nonusers" were people with no prescription for either type of medication in the prior 365 days.

Information about covariates came from electronic data and detailed medical record review. Medical record reviewers were blinded to case-control status. The reviews focused on the 2 year period prior to September 1 of each year (defined hereafter as the baseline period) and assessed the presence and severity of various health conditions believed to be associated with pneumonia risk. The time frame for medical record review was chosen based on the aims of the original study, in which health status at the time of influenza vaccination was of particular interest. Reviewers categorized the severity of comorbid illnesses using measures such as need for home oxygen, long-term corticosteroid use for lung disease, and measurement of ejection fraction (EF). Functional status measures were defined based on documentation in the chart and included need for assistance with ambulation or bathing, use of home health services, and whether a subject was ever described as frail by medical personnel. Cognitive status was defined based on the presence of a physician diagnosis of dementia in the chart. Computerized pharmacy data provided information about other medications expected to be associated with pneumonia risk, including medications for chronic lung or heart disease. We obtained information about health conditions for which PPI and H2 blockers are commonly prescribed from ICD-9 codes from inpatient and outpatient encounters.

\section{Analysis}

We descriptively characterized factors associated with pneumonia risk, calculating proportions for categorical variables and medians and interquartile ranges for continuous variables. Among control subjects, we examined characteristics associated with current use of PPIs or $\mathrm{H} 2$ blockers. Conditional logistic regression was used to estimate adjusted odds ratios (ORs) and 95\% confidence intervals (CIs) for the association between current use of PPIs or $\mathrm{H} 2$ blockers and pneumonia risk. The referent group was people who did not use either medication class. We selected potential confounders for our final models a priori, based on review of the literature and clinical plausibility, focusing on conditions known to be associated with pneumonia. We included markers of severity of comorbid illness and measures of functional and cognitive status. To ensure that no unexpected potential confounders were omitted, we also examined the results of the descriptive analyses described above for unexpected associations.

We assessed the robustness of our results in sensitivity analyses. First, we applied a stricter definition of "current use" based on whether the case's date of pneumonia onset fell within the expected duration of the most recent prescription (multiplying by 125\% to allow for imperfect compliance.) Second, to examine the impact of covariate selection, we constructed a parsimonious model that included only those covariates whose inclusion altered the main risk estimate by $10 \%$ or more. Third, we examined PPI use separately from $\mathrm{H} 2$ blocker use. Fourth, we stratified by pneumonia severity (ambulatory versus hospitalized). Finally, because in prior studies pneumonia risk varied according to the recency of initiating acidsuppressing medications, ${ }^{14}, 18$ we stratified by recency of initiation ( $<30$ days, $\geq 30$ days). Initiation was defined as filling a prescription after a gap of at least 60 days since any prior prescription would have been consumed (allowing for imperfect compliance.) Except for the second sensitivity analysis (the parsimonious model), these analyses adjusted for the same covariates as the primary analysis.

Most prior studies of this topic used automated data only, without medical record review to validate cases or identify covariates. So we examined the impact of aspects of our study design including validation of cases, restriction of the cohort, and ascertainment of confounders from medical records by carrying out analyses using only administrative and 
pharmacy data. We conducted a nested case-control study within the source population of older adults age 65-94 enrolled in GH during the study time frame. We excluded nursing home residents because of incomplete information about their medication use. For different models, pneumonia cases were either presumptive cases (identified from ICD-9 codes) or validated cases (based on medical record review.) Using incidence density sampling, we selected up to 3 controls per case, matched on age, sex, and calendar year. The exposure (current use of PPIs or H2 blockers) was identified from pharmacy data and defined as in our primary analyses. Analyses used multivariable conditional logistic regression modeling. The first model included cases identified from ICD-9 codes with adjustment for age, sex, and the following covariates defined from administrative $\mathrm{e}^{25}$ and pharmacy data: chronic pulmonary disease, congestive heart failure, cerebrovascular disease, hemi- or paraplegia, myocardial infarction, dementia, renal disease, malignancy, moderate or severe liver disease, diabetes, number of outpatient visits, and use of inhaled bronchodilators or inhaled or oral corticosteroids. Model 1 is intended to most closely resemble the analyses carried out in prior studies. For the second model, we used administrative and pharmacy data to restrict the population, excluding people with malignancy, renal disease, or use of immunosuppressive medications. In a third model, we did not restrict the population but examined only validated pneumonia cases and their matched controls. Fourth, we applied both restriction and validation. Models 2 through 4 adjusted for the same covariates as Model 1. Analyses were carried out in SAS version 9.1 (SAS Institute, Incorporated, Cary, North Carolina, United States).

\section{Results}

We identified 1125 cases of CAP occurring from 2000-2003 and 2235 matched controls. The median age was 77, and about half were male. Among CAP cases, 395 (35.1\%) were hospitalized, and $62(5.5 \%)$ died within 30 days of their index date. Ranitidine was the most commonly used acid-suppressing medication, accounting for $45 \%$ of prescriptions, followed by pantoprazole, accounting for $35 \%$. Appendix Table 2 (online) details the specific medications used.

People with pneumonia were more likely than controls to have comorbid illnesses, particularly chronic lung or heart disease, and to have more severe disease (Table 2). They were also more likely than controls to have functional or cognitive impairment. People currently using PPIs and $\mathrm{H} 2$ blockers were more likely than nonusers to have chronic lung disease or congestive heart failure, to have indicators of more severe disease, and to have functional impairment (Table 3).

Among people with pneumonia, 21.4\% (241/1125) were current users of PPIs or H2 blockers compared to $15.7 \%$ (350/2235) of controls (Table 4). The OR adjusted only for matching variables was 1.56 (95\% CI, 1.34-1.81) comparing current use to nonuse, but further adjustment resulted in an OR close to one (adjusted OR 1.03, 95\% CI 0.86-1.24). The three confounders whose inclusion in the model most altered the main exposure odds ratio were chronic obstructive pulmonary disease, the number of outpatient visits in the prior year, and use of inhaled corticosteroids. A higher risk of CAP was seen in possible users compared to nonusers.

The null result for current use was unchanged in sensitivity analyses using a more stringent definition for "current use," adjusting for only the most influential covariates (the parsimonious model), or stratifying by pneumonia severity (Figure 2). The slightly increased CAP risk seen with "possible" use was again observed with the more stringent definition of current use (adjusted OR 1.25, 95\% CI 1.00-1.55). No increased pneumonia risk was seen when current use was stratified by recency of initiation. The risk of CAP was not 
appreciably elevated in current users of PPIs (adjusted OR 1.13, 95\% CI 0.88-1.44, compared to nonuse).

In administrative-data analyses that defined pneumonia based solely on ICD-9 codes and adjusted for age, gender, and covariates from administrative and pharmacy data, the OR for current use of a PPI or H2 blocker compared to nonuse was 1.19 (95\% CI 1.09-1.30), and for current use of a PPI, 1.32 (1.17-1.49). These estimates changed little with restriction of the population using administrative and pharmacy data (ORs 1.18 and 1.36, respectively). Results were substantially farther from the null when only validated pneumonia cases were included (OR for PPI or H2 blocker use, 1.33 [1.16-1.53], and for PPI use, 1.61 [1.331.94].) Results for the model with both validation and restriction were similar to the model with validation alone.

\section{Discussion}

\section{Principal findings}

We observed no increased pneumonia risk with current use of PPIs or H2 blockers in community-dwelling, immunocompetent older adults. The odds of pneumonia was 1.13 (95\% CI 0.88-1.44) comparing current PPI use to nonuse and 1.03 [0.86-1.24] comparing use of either medication class to nonuse. Our results were robust to sensitivity analyses, and pneumonia risk did not differ according to recency of initiating these medications.

An unexpected finding was a slight increase in CAP risk associated with "possible use" of these medications (OR 1.28, 95\% CI 1.03-1.59), which was defined as filling at least one prescription in the past year but not 2 in the past 6 months. This category of use probably reflects past or sporadic use of these medications rather than ongoing, sustained use. We are not aware of a biologic mechanism to explain increased risk only with past or sporadic use but not with ongoing use. Although this "possible use" group likely includes some new initiators, we conducted sensitivity analyses that explicitly examined recency of initiation and observed no elevation in risk for recent initiation. Thus the results observed for "possible use" are unlikely to be due to increased risk among new users. It may be that this pattern of use indicates nonadherence, which in numerous studies (including studies of placebo) has been associated with poor health outcomes. ${ }^{27,} 28$ People who are nonadherent to prescribed medications often have worse health status or health habits than those who are adherent. $^{29}$

\section{Comparison with prior studies}

Analyses summarizing RCT results have had limited ability to examine pneumonia risk in relation to use of acid-suppressing medications. ${ }^{12,13}$ The small number of cases in these analyses resulted in imprecise risk estimates that were consistent with no association or with clinically important harm (Table 1). Results of prior observational studies are inconsistent. Most $^{14-17}$ but not all ${ }^{18}$ studies observed increased risk with current PPI use compared to past use or nonuse. Only 1 study observed increased risk with use of $\mathrm{H} 2$ blockers. ${ }^{16}$ Of the 4 prior studies that reported increased pneumonia risk with PPI use, three examined a time period similar to ours. ${ }^{14,} 16,17$ In all prior studies, most cases of pneumonia occurred in older adults. Thus, variation in the time period or age of the population studied is not likely to explain the discrepant results. ${ }^{14}, 16-18$

The most substantial differences between our study and others relate to methodology. Unlike most prior studies, we validated pneumonia cases. We restricted analyses to immunocompetent community-dwelling older adults, which should decrease confounding due to health status and frailty. Ours is the first study of this topic to use detailed medical record review to ascertain confounders. Medical record review is more accurate than 
administrative data for many health conditions. ${ }^{19,}{ }^{20}$ Important confounders in our study included chronic lung and heart disease, which may be poorly measured in administrative data. ${ }^{19}$ The impact of these methodologic steps can be seen from sensitivity analyses which used administrative and pharmacy data only. These analyses yielded risk estimates that were higher than those from our primary analyses, e.g. OR of 1.32 [1.17-1.49] for pneumonia among PPI users compared to nonusers, increasing to 1.61 [1.33-1.94] when only validated cases were examined. These estimates contrast with our primary study results of 1.13 [0.881.44] comparing current PPI use to nonuse. Taken by themselves, the administrative-data analyses would likely have been interpreted as showing increased pneumonia risk in current users of these medications. The discrepancy between our primary case-control analysis and the administrative-data sensitivity analysis probably reflects residual confounding in the administrative-data analysis because administrative data have poor sensitivity for many important health conditions associated with pneumonia. For example, in one study the sensitivity of administrative data was only $13 \%$ for pulmonary disease, ${ }^{19}$ while in another, it ranged from $22-62 \%$ depending on the algorithm used. ${ }^{30}$ Inaccurate classification of confounder status can result in residual confounding. We believe that our primary results, which are based on extensive medical record review as well as automated data, are more valid than the administrative-data analyses due to the extensive efforts to address confounding and the greater accuracy of the data in many regards.

In prior studies that stratified by recency of initiation, the association of $\mathrm{H} 2$ blocker or PPI use with pneumonia risk was strongest with very recent initiation and weaker or absent for use of long duration. ${ }^{14,}$ 16, 18 This was true for both PPIs and H2 blockers. ${ }^{16,}{ }^{18}$ For instance, Sarkar et al. observed a nearly 7-fold increased risk within 2 days of initiating PPI use, but no increased risk 30 days or more after initiation. ${ }^{18}$ There was a similarly dramatic increase for very recent initiation of $\mathrm{H} 2$ blockers. This temporal pattern is not consistent with the proposed biological mechanism by which these medications might increase pneumonia risk. Neither gastric acid suppression nor bacterial colonization would be expected to occur so rapidly, nor to wane with continued use. Herzig et al. suggested an alternative mechanism, acute immunosuppressive effects, ${ }^{15}$ citing immunologic studies in which exposure to PPIs impaired immune cell function. ${ }^{31,} 32$ However, this mechanism does not explain why no elevated risk is seen with long term use, and it seems unlikely that both $\mathrm{H} 2$ blockers and PPIs would have the same acute effect on immune function. In fact, one immunologic study reported that $\mathrm{H} 2$ blockers did not impair immune cell function. ${ }^{33}$ The pattern observed by Sarkar et al. is more consistent with bias due to confounding or reverse causation. Confounding could occur if a PPI is prescribed at the time of a change in health status (e.g., when a potentially hazardous medication is initiated), while reverse causation could occur if the PPI were initiated for early symptoms of pneumonia.

\section{Limitations of the study}

There may be misclassification of exposure status if people obtained $\mathrm{H} 2$ blockers OTC at non-GH pharmacies or filled prescriptions for acid-suppressing medications at $\mathrm{GH}$ pharmacies but did not actually take them. H2 blockers were available OTC during the study period, but PPIs were not. Evidence suggests that despite the OTC availability of $\mathrm{H} 2$ blockers, GH pharmacy data captured the majority of use of acid-suppressing medications by older adults during this time period. ${ }^{24} \mathrm{We}$ included pneumonia cases occurring during 8 or 9 months of each study year, rather than the entire year. This could introduce bias if the association between acid-suppressing medications and pneumonia risk varies by season. However, the majority of pneumonia cases occur during influenza season, which was included in the study time frame. There may be residual confounding by unmeasured confounders. Our sample size did not permit us to conduct analyses that were finely stratified by recency of initiation nor to examine individual acid-suppressing medications. 
We lack power to rule out a modest increase in pneumonia risk associated with PPI use. However, it is noteworthy that our more rigorous methods yielded a much smaller point estimate than prior studies with positive findings, ${ }^{14-17}$ while results from our administrative-data analyses (designed to be comparable to prior studies) yielded risk estimates that were further from the null. It remains challenging for observational studies to provide credible information about very small increases in risk, given the trade-off between precision (requiring large sample size) and validity (requiring detailed, accurate data.)

\section{Future research directions}

The conflicting results from studies of this question highlight the challenges of using administrative data to study adverse effects of medication use in large populations. Medical record review provides better information but at substantial cost. Several design and analysis methods offer promise for improving validity at lower cost, including two-stage sampling, ${ }^{34}$ restriction of the study sample, and thoughtful choice of the comparator group. ${ }^{35}$ There is a need for further research to improve the validity of results obtained from automated data.

\section{Conclusion}

While some prior studies reported increased risk of pneumonia associated with current use of acid-suppressing medications, particularly PPIs, we observed no such increase in our study which collected and adjusted for more detailed and accurate information about pneumonia outcomes and confounding factors. Taken as a whole, the current evidence does not support a substantial increased pneumonia risk associated with use of acid-suppressive medications.

\section{Key points}

- Use of PPIs and $\mathrm{H} 2$ blockers is not associated with increased risk of community-acquired pneumonia in older adults.

- Increased pneumonia risk was not seen with recent initiation of these medications.

- In contrast to analyses using detailed clinical data, analyses using only administrative and pharmacy data yielded elevated risk estimates, suggesting residual confounding.

- Important confounders included chronic lung disease and markers of disease severity, which may not be captured well in administrative data.

\section{Acknowledgments}

Financial support: Dr. Dublin was funded by a Paul Beeson Career Development Award from the National Institute on Aging, grant K23AG028954. The Beeson Award is also supported by the American Federation for Aging Research and the Hartford and Starr Foundations and Atlantic Philanthropies. Group Health Research Institute internal funds covered the data collection and analysis. The content is solely the responsibility of the authors and does not necessarily represent the official views of the National Institute on Aging or the National Institutes of Health.

The authors thank Dr. Gregory E. Simon, MD, MPH, for his helpful review of the manuscript. 


\section{References}

1. Shaheen NJ, Hansen RA, Morgan DR, et al. The burden of gastrointestinal and liver diseases, 2006. Am J Gastroenterol. 2006; 101:2128-38. AJG723 [pii]. 10.1111/j.1572-0241.2006.00723.x [PubMed: 16848807]

2. Parente F, Cucino C, Gallus S, et al. Hospital use of acid-suppressive medications and its fall-out on prescribing in general practice: a 1-month survey. Aliment Pharmacol Ther. 2003; 17:1503-6. 1600 [pii]. [PubMed: 12823152]

3. Zink DA, Pohlman M, Barnes M, et al. Long-term use of acid suppression started inappropriately during hospitalization. Aliment Pharmacol Ther. 2005; 21:1203-9. APT2454 [pii]. 10.1111/j.13652036.2005.02454.x [PubMed: 15882240]

4. Nardino RJ, Vender RJ, Herbert PN. Overuse of acid-suppressive therapy in hospitalized patients. Am J Gastroenterol. 2000; 95:3118-22. S0002-9270(00)02052-9 [pii]. 10.1111/j.15720241.2000.03259.x [PubMed: 11095327]

5. Bashford JN, Norwood J, Chapman SR. Why are patients prescribed proton pump inhibitors? Retrospective analysis of link between morbidity and prescribing in the General Practice Research Database. BMJ. 1998; 317:452-6. [PubMed: 9703528]

6. George CJ, Korc B, Ross JS. Appropriate proton pump inhibitor use among older adults: a retrospective chart review. Am J Geriatr Pharmacother. 2008; 6:249-54. S1543-5946(08)00072-X [pii]. 10.1016/j.amjopharm.2008.12.001 [PubMed: 19161927]

7. Glew CM, Rentler RJ. Use of proton pump inhibitors and other acid suppressive medications in newly admitted nursing facility patients. J Am Med Dir Assoc. 2007; 8:607-9. S15258610(07)00340-4 [pii]. 10.1016/j.jamda.2007.07.001 [PubMed: 17998118]

8. Heidelbaugh JJ, Inadomi JM. Magnitude and economic impact of inappropriate use of stress ulcer prophylaxis in non-ICU hospitalized patients. Am J Gastroenterol. 2006; 101:2200-5. AJG839 [pii]. 10.1111/j.1572-0241.2006.00839.x [PubMed: 16952283]

9. Kantorova I, Svoboda P, Scheer P, et al. Stress ulcer prophylaxis in critically ill patients: a randomized controlled trial. Hepato-Gastroenterology. 2004; 51:757-61. [PubMed: 15143910]

10. Prod'hom G, Leuenberger P, Koerfer J, et al. Nosocomial pneumonia in mechanically ventilated patients receiving antacid, ranitidine, or sucralfate as prophylaxis for stress ulcer. A randomized controlled trial. Ann Intern Med. 1994; 120:653-62. [PubMed: 8135449]

11. Williams C. Occurrence and significance of gastric colonization during acid-inhibitory therapy. Best Pract Res Clin Gastroenterol. 2001; 15:511-21. [PubMed: 11403543]

12. Sultan N, Nazareno J, Gregor J. Association between proton pump inhibitors and respiratory infections: a systematic review and meta-analysis of clinical trials. Can J Gastroenterol. 2008; 22:761-6. [PubMed: 18818790]

13. Estborn L, Joelson S. Occurrence of community-acquired respiratory tract infection in patients receiving esomeprazole: retrospective analysis of adverse events in 31 clinical trials. Drug Saf. 2008; 31:627-36. 3178 [pii]. [PubMed: 18558796]

14. Gulmez SE, Holm A, Frederiksen H, et al. Use of proton pump inhibitors and the risk of community-acquired pneumonia: a population-based case-control study. Arch Intern Med. 2007; 167:950-5. 167/9/950 [pii]. 10.1001/archinte.167.9.950 [PubMed: 17502537]

15. Herzig SJ, Howell MD, Ngo LH, et al. Acid-suppressive medication use and the risk for hospitalacquired pneumonia. JAMA. 2009; 301:2120-8. 301/20/2120 [pii]. 10.1001/jama.2009.722 [PubMed: 19470989]

16. Laheij RJ, Sturkenboom MC, Hassing RJ, et al. Risk of community-acquired pneumonia and use of gastric acid-suppressive drugs. JAMA. 2004; 292:1955-60. [PubMed: 15507580]

17. Myles PR, Hubbard RB, McKeever TM, et al. Risk of community-acquired pneumonia and the use of statins, ace inhibitors and gastric acid suppressants: a population-based case-control study. Pharmacoepidemiol Drug Saf. 2009; 18:269-75.10.1002/pds.1715 [PubMed: 19235776]

18. Sarkar M, Hennessy S, Yang YX. Proton-pump inhibitor use and the risk for community-acquired pneumonia. Ann Intern Med. 2008; 149:391-8. 149/6/391 [pii]. [PubMed: 18794558] 
19. Ahmed F, Janes GR, Baron R, et al. Preferred provider organization claims showed high predictive value but missed substantial proportion of adults with high-risk conditions. J Clin Epidemiol. 2005; 58:624-8. [PubMed: 16028341]

20. Quan H, Parsons GA, Ghali WA. Validity of information on comorbidity derived from ICD-9CCM administrative data. Med Care. 2002; 40:675-85. [PubMed: 12187181]

21. Jackson ML, Nelson JC, Weiss NS, et al. Influenza vaccination and risk of community-acquired pneumonia in immunocompetent elderly people: a population-based, nested case-control study. Lancet. 2008; 372:398-405. S0140-6736(08)61160-5 [pii]. 10.1016/S0140-6736(08)61160-5 [PubMed: 18675690]

22. Nelson JC, Jackson M, Yu O, et al. Impact of the introduction of pneumococcal conjugate vaccine on rates of community acquired pneumonia in children and adults. Vaccine. 2008; 26:4947-54. S0264-410X(08)00933-X [pii]. 10.1016/j.vaccine.2008.07.016 [PubMed: 18662735]

23. Saunders, KW.; Davis, RL.; Stergachis, A. Group Health Cooperative. In: Strom, BL., editor. Pharmacoepidemiology. Chichester: John Wiley \& Sons; 2005. p. 234

24. Boudreau DM, Doescher MP, Saver BG, et al. Reliability of Group Health Cooperative automated pharmacy data by drug benefit status. Pharmacoepidemiol Drug Saf. 2005; 14:877-84. [PubMed: 15931653]

25. Deyo RA, Cherkin DC, Ciol MA. Adapting a clinical comorbidity index for use with ICD-9-CM administrative databases. J Clin Epidemiol. 1992; 45:613-9. 0895-4356(92)90133-8 [pii]. [PubMed: 1607900]

26. Schoenfeld D. Partial residuals for the proportional hazards regression model. Biometrika. 1982; 69:239-41.

27. Simpson SH, Eurich DT, Majumdar SR, et al. A meta-analysis of the association between adherence to drug therapy and mortality. BMJ. 2006; 333(15) bmj.38875.675486.55 [pii]. 10.1136/bmj.38875.675486.55

28. Influence of adherence to treatment and response of cholesterol on mortality in the coronary drug project. N Engl J Med. 1980; 303:1038-41. [PubMed: 6999345]

29. Brookhart MA, Patrick AR, Dormuth C, et al. Adherence to lipid-lowering therapy and the use of preventive health services: an investigation of the healthy user effect. Am J Epidemiol. 2007; 166:348-54. [PubMed: 17504779]

30. Rector TS, Wickstrom SL, Shah M, et al. Specificity and sensitivity of claims-based algorithms for identifying members of Medicare+Choice health plans that have chronic medical conditions. Health Serv Res. 2004; 39:1839-57. HESR321 [pii]. 10.1111/j.1475-6773.2004.00321.x [PubMed: 15533190]

31. Zedtwitz-Liebenstein K, Wenisch C, Patruta S, et al. Omeprazole treatment diminishes intra- and extracellular neutrophil reactive oxygen production and bactericidal activity. Crit Care Med. 2002; 30:1118-22. [PubMed: 12006811]

32. Alkim H, Unal S, Okur H, et al. Omeprazole inhibits natural killer cell functions. Dig Dis Sci. 2008; 53:347-51.10.1007/s10620-007-9869-2 [PubMed: 17597410]

33. Mikawa K, Akamatsu H, Nishina K, et al. The effects of cimetidine, ranitidine, and famotidine on human neutrophil functions. Anesth Analg. 1999; 89:218-24. [PubMed: 10389808]

34. Breslow NE, Chatterjee N. Design and analysis of two-phase studies with binary outcomes applied to Wilms tumor prognosis. Applied Statistics. 1999; 48:457-68.

35. Setoguchi S, Glynn RJ, Avorn J, et al. Statins and the risk of lung, breast, and colorectal cancer in the elderly. Circulation. 2007; 115:27-33. CIRCULATIONAHA.106.650176 [pii] 10.1161. [PubMed: 17179016] 


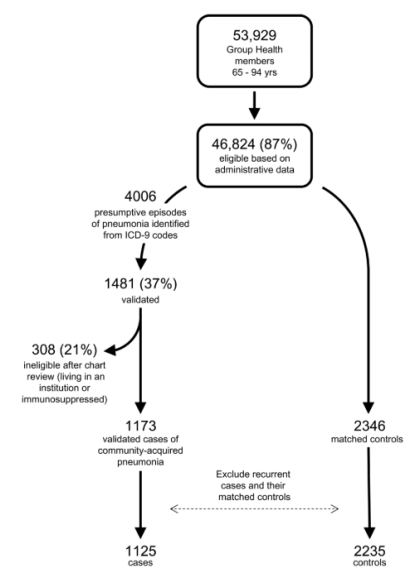

Figure 1.

Selection of cases and controls for inclusion in the study 


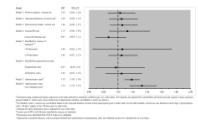

Figure 2.

Risk estimates for the association between PPI or $\mathrm{H} 2$ blocker use and community-acquired pneumonia: sensitivity analyses 


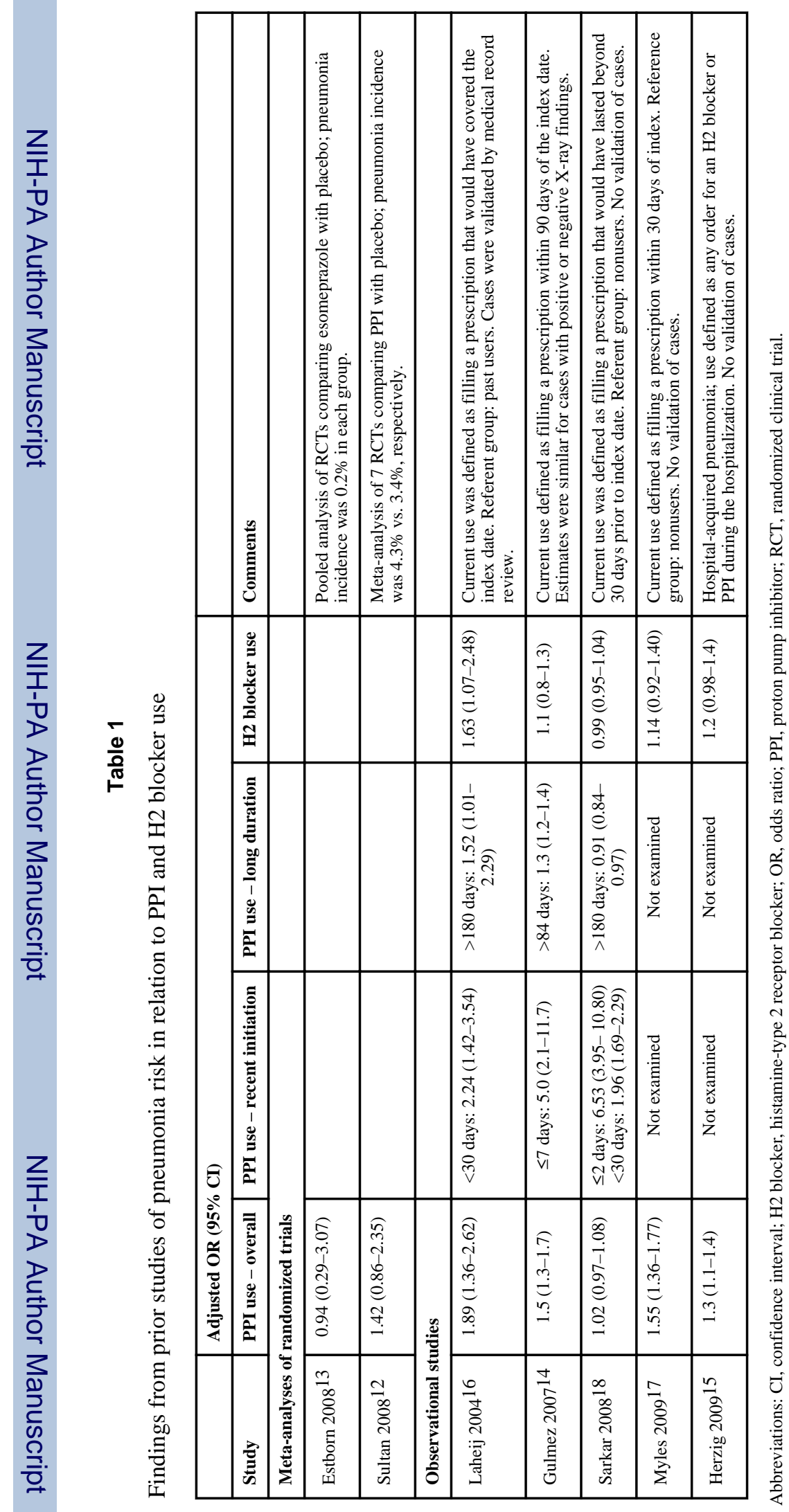


Table 2

Characteristics of pneumonia cases and controls

\begin{tabular}{|c|c|c|}
\hline & Pneumonia cases $(\mathrm{N}=1,125)$ & Controls $(\mathrm{N}=\mathbf{2 , 2 3 5})$ \\
\hline Characteristic $^{*}$ & $\mathbf{n}(\%)^{\dagger}$ & $\mathbf{n}(\%)^{\dagger}$ \\
\hline Median age (IQR), years & $77(71-82)$ & $77(71-82)$ \\
\hline Male & $571(50.8)$ & $1135(50.8)$ \\
\hline Median BMI (IQR), $\mathrm{kg} / \mathrm{m}^{2 \dagger}$ & $\begin{array}{c}26.5(23.4-30.6) \\
\quad(\mathrm{N}=799)\end{array}$ & $\begin{array}{c}27.3(24.5-30.6) \\
\quad(\mathrm{N}=1481)\end{array}$ \\
\hline Current smoker & $103 / 1109(9.2)$ & $115 / 2182(5.1)$ \\
\hline Asthma & $195(17.3)$ & $176(7.9)$ \\
\hline Chronic obstructive pulmonary disease (COPD) & $350(31.1)$ & $234(10.5)$ \\
\hline Hospitalized for COPD & $53(4.7)$ & $15(0.7)$ \\
\hline Home oxygen use & $92(8.2)$ & $21(0.9)$ \\
\hline Long term steroid use for lung disease & $29(2.6)$ & $8(0.4)$ \\
\hline FEV1 measured & $179(15.9)$ & $86(3.8)$ \\
\hline Congestive heart failure (CHF) & $207(18.4)$ & $156(7.0)$ \\
\hline Hospitalized for CHF & $34(3.0)$ & $18(0.8)$ \\
\hline Ejection fraction measured & $79(7.0)$ & $42(1.9)$ \\
\hline Myocardial infarction & $137(12.2)$ & $217(9.7)$ \\
\hline Coronary revascularization & $155(13.8)$ & $250(11.2)$ \\
\hline Stroke & $93(8.3)$ & $153(6.8)$ \\
\hline Swallowing disorder leading to aspiration & $17(1.5)$ & $11(0.5)$ \\
\hline Alcoholism & $17(1.5)$ & $24(1.1)$ \\
\hline Diabetes mellitus & $194(17.2)$ & $337(15.1)$ \\
\hline Dementia & $53(4.7)$ & $75(3.4)$ \\
\hline Gastroesophageal reflux disease ${ }^{\S}$ & $172(15.3)$ & $285(12.8)$ \\
\hline Peptic ulcer ${ }^{\S}$ & $46(4.1)$ & $46(2.1)$ \\
\hline Dyspepsia ${ }^{\S}$ & $74(6.6)$ & $98(4.4)$ \\
\hline At least one functional impairment & $206(18.3)$ & $261(11.7)$ \\
\hline Requires assistance bathing & $24(2.1)$ & $15(0.7)$ \\
\hline Requires assistance walking & $198(17.6)$ & $250(11.2)$ \\
\hline Any use of home health services & $161(14.3)$ & $137(6.1)$ \\
\hline Frailll & $88(7.8)$ & $64(2.9)$ \\
\hline Bronchodilator use ${ }^{I}$ & $147(13.1)$ & $60(2.7)$ \\
\hline Furosemide use ${ }^{q l}$ & $254(22.6)$ & $228(10.2)$ \\
\hline Inhaled corticosteroid use ${ }^{I l}$ & $258(22.9)$ & $175(7.8)$ \\
\hline Insulin or oral hypoglycemic use $\mathrm{I}^{\mathscr{I}}$ & $129(11.5)$ & $209(9.4)$ \\
\hline Oral corticosteroid use ${ }^{\text {II }}$ & $174(15.5)$ & $111(5.0)$ \\
\hline Received pneumococcal vaccine ${ }^{* *}$ & $1031(91.6)$ & $2046(91.5)$ \\
\hline Received influenza vaccine ${ }^{\dagger \dagger}$ & $681(60.5)$ & $1374(61.5)$ \\
\hline
\end{tabular}




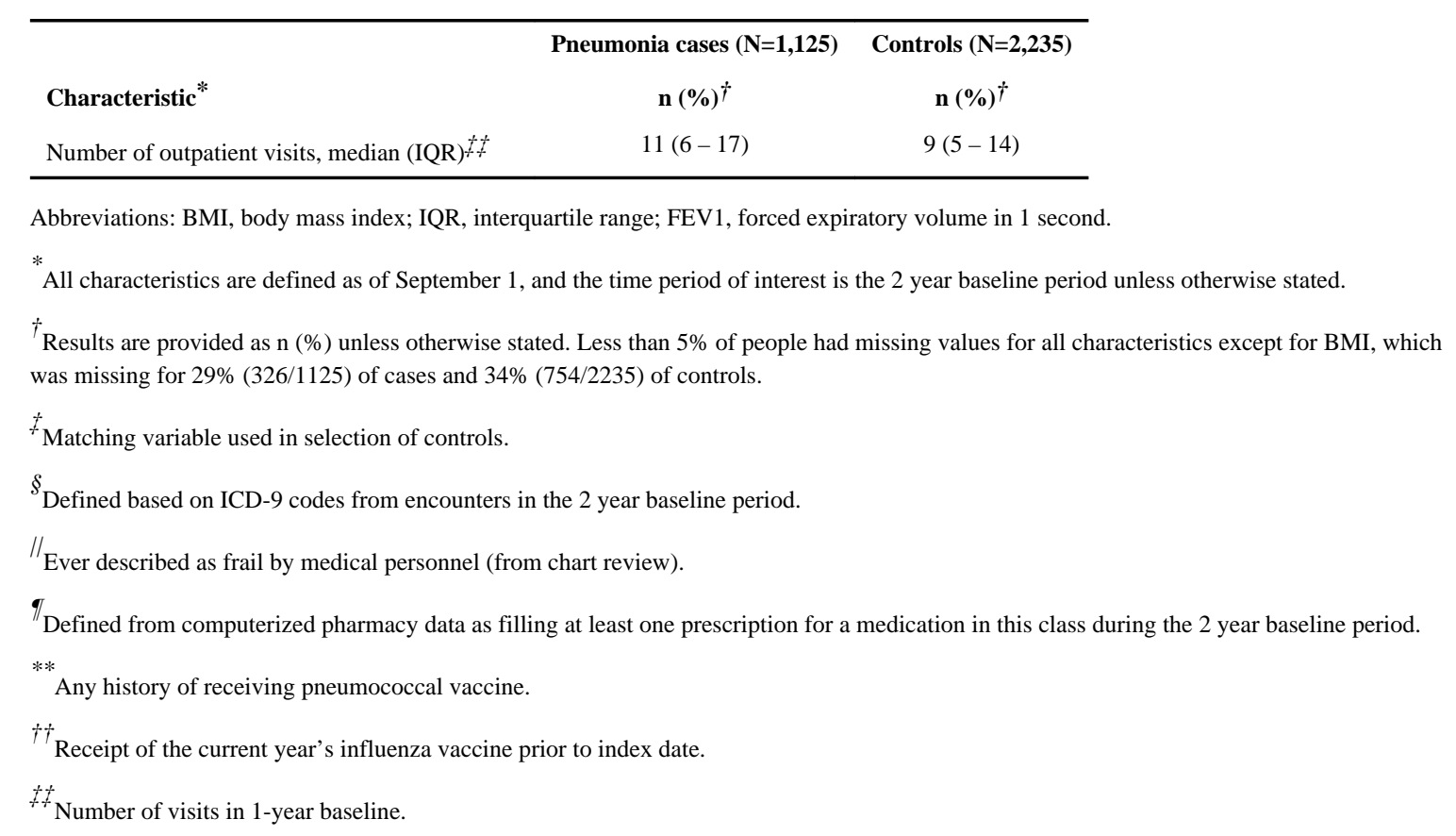


Table 3

Characteristics associated with use of PPIs and $\mathrm{H} 2$ blockers among controls

\begin{tabular}{|c|c|c|}
\hline & Nonusers* $(N=1670)$ & Current users* $^{*}(\mathrm{~N}=\mathbf{3 5 0})$ \\
\hline Characteristic $^{\dagger}$ & $\mathbf{n}(\%)^{*}$ & $\mathrm{n}(\%)^{*}$ \\
\hline Age, median (IQR) & $77(71-82)$ & $77(71-81)$ \\
\hline Male & $866(51.9)$ & $163(46.6)$ \\
\hline BMI, median (IQR) $)^{*}$ & $\begin{array}{c}26.9(24.0-30.6) \\
\quad(\mathrm{N}=1096)\end{array}$ & $\begin{array}{c}27.4(24.7-30.7) \\
\quad(\mathrm{N}=239)\end{array}$ \\
\hline Current smoker & $87 / 1622(5.4)$ & $17 / 345(4.9)$ \\
\hline Asthma & $106(6.3)$ & $49(14.0)$ \\
\hline Chronic obstructive pulmonary disease & $148(8.9)$ & $62(17.7)$ \\
\hline Hospitalized for COPD & $10(0.6)$ & $4(1.1)$ \\
\hline Home oxygen use & $12(0.7)$ & $8(2.3)$ \\
\hline Long term steroid use for lung disease & $2(0.1)$ & $5(1.4)$ \\
\hline FEV1 ever measured & $46(2.8)$ & $29(8.3)$ \\
\hline Congestive heart failure & $106(6.3)$ & $36(10.2)$ \\
\hline Hospitalized for $\mathrm{CHF}$ & $13(0.8)$ & $4(1.1)$ \\
\hline Ejection fraction measured & $22(1.3)$ & $15(4.3)$ \\
\hline Myocardial infarction & $135(8.1)$ & $52(14.9)$ \\
\hline Coronary revascularization & $162(9.7)$ & $51(14.6)$ \\
\hline Stroke & $105(6.3)$ & $37(10.6)$ \\
\hline Swallowing disorder leading to aspiration & $4(0.2)$ & $5(1.4)$ \\
\hline Alcoholism & $20(1.2)$ & $1(0.3)$ \\
\hline Diabetes mellitus & $251(15.0)$ & $57(16.3)$ \\
\hline Dementia & $59(3.5)$ & $12(3.4)$ \\
\hline Gastroesophageal reflux disease $\S$ & $70(4.2)$ & $156(44.6)$ \\
\hline Peptic ulcer $\S$ & $13(0.8)$ & $20(5.7)$ \\
\hline Dyspepsia $\S$ & $24(1.4)$ & 45 (12.9) \\
\hline At least one functional impairment & $177(10.6)$ & $57(16.3)$ \\
\hline Requires assistance bathing & $10(0.6)$ & $4(1.1)$ \\
\hline Requires assistance walking & $168(10.1)$ & $55(15.7)$ \\
\hline Any use of home health services & $92(5.5)$ & $29(8.3)$ \\
\hline Fraill & $43(2.6)$ & $13(3.7)$ \\
\hline Bronchodilator use $^{I I}$ & $34(2.0)$ & $19(5.4)$ \\
\hline Furosemide use ${ }^{I I}$ & $138(8.3)$ & $66(18.9)$ \\
\hline Inhaled corticosteroid use $\mathrm{e}^{q}$ & $101(6.0)$ & $55(15.7)$ \\
\hline Insulin or oral hypoglycemic use ${ }^{I l}$ & $152(9.1)$ & $38(10.9)$ \\
\hline Oral corticosteroid use $\mathrm{I}^{\mathscr{I}}$ & $65(3.9)$ & $32(9.1)$ \\
\hline Received pneumococcal vaccine ${ }^{* *}$ & $1,519(91.0)$ & $332(94.9)$ \\
\hline Received influenza vaccine ${ }^{\dagger \dagger}$ & $1,020(61.1)$ & $225(64.3)$ \\
\hline
\end{tabular}




\begin{tabular}{lcc}
\hline & Nonusers* $^{*}(\mathbf{N}=\mathbf{1 6 7 0})$ & Current users* ${ }^{*}(\mathbf{N}=\mathbf{3 5 0})$ \\
Characteristic $^{\dagger}$ & $\mathbf{n}(\boldsymbol{\%})^{*}$ & $\mathbf{n}(\boldsymbol{\%})^{*}$ \\
Number of outpatient visits, median $(\mathrm{IQR})^{+\neq}$ & $8(5-14)$ & $13(8-21)$ \\
\hline
\end{tabular}

Abbreviations: BMI, body mass index; IQR, interquartile range; FEV1, forced expiratory volume in 1 second; H2 blocker, histamine-type 2 receptor blocker; PPI, proton pump inhibitor.

* Current use is defined as receiving $\geq 2$ fills of a PPI and/or H2 blocker, each with $\geq 30$ days' supply, within the 180 days prior to index date. Nonusers had no fills for a PPI or H2 blocker in the 365 days prior to index date.

${ }^{\dagger}$ All characteristics are defined as of September 1, and the time period of interest is the 2 year baseline period unless otherwise stated.

* and $32 \%(111 / 350)$ of current users.

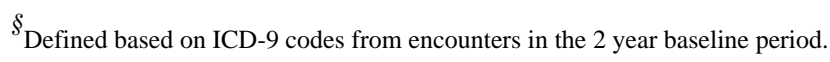

$\|_{\text {Ever described as frail by medical personnel (from chart review). }}$

${ }^{I}$ Defined from computerized pharmacy data as filling at least one prescription for a medication in this class during the 2 year baseline period. ** Any history of receiving pneumococcal vaccine.

${ }^{\dagger \dagger}$ Receipt of the current year's influenza vaccine prior to index date.

Number of visits in 1-year baseline. 


\section{Table 4}

Risk of CAP in relation to use of PPIs and $\mathrm{H} 2$ blockers

\begin{tabular}{lllll}
\hline & Cases $(\mathbf{N = 1 , 1 2 5})$ & Controls $(\mathbf{N = 2 , 2 3 5})$ & \multicolumn{2}{l}{ Odds Ratio (95\% CI) } \\
PPI/H2 blocker use & $\mathbf{n}(\%)$ & $\mathbf{n}(\%)$ & Minimally adjusted $^{\dagger}$ & Fully adjusted $^{*}$ \\
No use & $741(65.9)$ & $1670(74.7)$ & $1.00($ Ref. $)$ & $1.00($ Ref.) \\
Possible use & $143(12.7)$ & $215(9.6)$ & $1.52(1.26,1.82)$ & $1.28(1.03,1.59)$ \\
Current use & $241(21.4)$ & $350(15.7)$ & $1.56(1.34,1.81)$ & $1.03(0.86,1.24)$ \\
\hline
\end{tabular}

Abbreviations: CAP, community-acquired pneumonia; CHF, congestive heart failure; CI, confidence interval; FEV 1 , forced expiratory volume in 1 second; H2 blocker, histamine type-2 receptor blocker; PPI, proton pump inhibitor; OR, odds ratio.

* Current use is defined as having $\geq 2$ fills of a PPI and/or H2 blocker, each with $\geq 30$ days' supply, within the 180 days prior to index date. Nonusers had no fills for a PPI or H2 blocker in the 365 days prior to index date. Possible users had at least 1 fill of a H2 blocker or PPI within the 365 days prior to index date but did not meet criteria for current use.

${ }^{\dagger}$ Adjusted only for matching variables (age, sex, index date).

*Adjusted for matching variables and for asthma, chronic obstructive pulmonary disease (COPD) and history of COPD hospitalization, use of home oxygen, long term steroid use for lung disease, FEV 1 measured, smoking status (current/recently quit, former, or never), congestive heart failure $(\mathrm{CHF})$ and history of $\mathrm{CHF}$ hospitalization, ejection fraction measured, stroke, myocardial infarction, coronary revascularization, other heart disease, swallowing disorder, alcoholism, pneumococcal or influenza vaccination, number of outpatient visits, need for assistance with ambulation or bathing, dementia, frailty, and use of the following medications: inhaled bronchodilators, inhaled corticosteroids, oral corticosteroids, or furosemide. 


\section{Appendix Table 1}

Inclusion and exclusion criteria (for online publication)

\begin{tabular}{|c|c|}
\hline Condition/criterion & Definition \\
\hline \multicolumn{2}{|l|}{ Inclusion criteria } \\
\hline Age criteria & Age $65-94$ \\
\hline Community dwelling & Not living in a nursing home or other institution \\
\hline Utilization & $\begin{array}{l}\geq 2 \text { GHC physician visits and/or home health visits in the year prior to September } 1 \text { (to ensure adequate } \\
\text { data available) }\end{array}$ \\
\hline \multicolumn{2}{|l|}{ Exclusion criteria } \\
\hline Chronic renal failure & $\begin{array}{l}\text { Administrative data: } \mathrm{ICD}-9 \text { codes } 403.1,404.2,404.3,586^{*} \\
\text { Laboratory data: most recent creatinine value prior to September } 1>3 \mathrm{mg} / \mathrm{dl} \\
\text { Chart review: }{ }^{*} \geq 1 \text { serum creatinine }>3 \mathrm{mg} / \mathrm{dL} \text { in the prior } 2 \text { years, or any dialysis, or a diagnosis of } \\
\text { chronic renal failure. }\end{array}$ \\
\hline Serious cancer ${ }^{*}$ & $\begin{array}{l}\text { Administrative data: ICD-9 codes } 150,151,155,157,158,159,162,163,191,196-202,203.0,203.1 \text {, } \\
\text { 204-208, or } 238.7 \\
\text { Chart review: Confirmed diagnosis of lung, trachea, bronchus, brain, esophagus, liver, pancreas, } \\
\text { peritoneum, pleura, or stomach cancer; or any mention of leukemia or lymphoma; or any mention of } \\
\text { myelodysplastic syndrome, multiple myeloma, lymphoproliferative neoplasm, or any metastatic cancer; } \\
\text { or any stage III or stage IV cancer regardless of site. }\end{array}$ \\
\hline Cancer treatment & Chemotherapy, radiation therapy, or surgery in the 3 months prior to September 1 of entry year. \\
\hline Immunosuppressive medications ${ }^{*}$ & $\begin{array}{l}\text { Computerized pharmacy data: any prescription for methotrexate, azathioprine, cyclosporine, } \\
\text { mycophenolate, tacrolimus, sirolimus, daclizumab, or muromonab-CD3 }\end{array}$ \\
\hline Human Immunodeficiency Virus* & Computerized pharmacy data: any prescription for medications for Human Immunodeficiency Virus \\
\hline Receiving hospice care & \\
\hline
\end{tabular}

*

in the two years prior to September 1 of entry year. 


\section{Appendix Table 2}

Use of PPIs and $\mathrm{H} 2$ blockers among study subjects* (for online publication)

\begin{tabular}{|l|c|c|}
\hline Acid-suppressing medication & $\begin{array}{c}\text { Number of prescriptions dispensed } \\
\mathbf{n}(\%)\end{array}$ & $\begin{array}{c}\text { Total days' supply dispensed } \\
\mathbf{n}(\%)\end{array}$ \\
\hline Total & 4,581 & 200,546 \\
PPI & & $68,552(34.2)$ \\
Pantoprazole & $1,584(34.6)$ & $12,836(6.4)$ \\
Lansoprazole & $307(6.7)$ & $9,579(4.8)$ \\
Omeprazole & $275(6.0)$ & \\
H2 blocker & & $92,333(46.0)$ \\
Ranitidine & $2,051(44.8)$ & $16,712(8.3)$ \\
Cimetidine & $353(7.7)$ & $534(0.3)$ \\
Famotidine & $11(0.2)$ & \\
\hline
\end{tabular}

Abbreviations: H2 blocker, histamine-type 2 receptor blocker; PPI, proton pump inhibitor.

* during the 365 days prior to index date. 